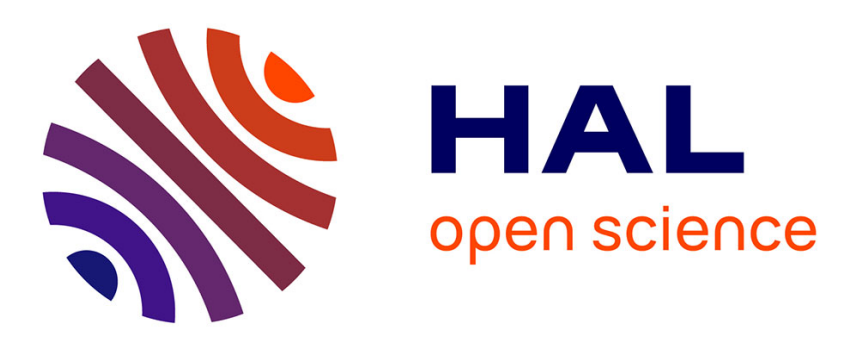

\title{
Multi-hop wireless charging optimization in Low-Power Networks
}

\author{
Tifenn Rault, Abdelmadjid Bouabdallah, Yacine Challal
}

\section{To cite this version:}

Tifenn Rault, Abdelmadjid Bouabdallah, Yacine Challal. Multi-hop wireless charging optimization in Low-Power Networks. IEEE Global Communications Conference, 2013, Atlanta, United States. pp.484-489. hal-00871198

\section{HAL Id: hal-00871198 \\ https://hal.science/hal-00871198}

Submitted on 9 Oct 2013

HAL is a multi-disciplinary open access archive for the deposit and dissemination of scientific research documents, whether they are published or not. The documents may come from teaching and research institutions in France or abroad, or from public or private research centers.
L'archive ouverte pluridisciplinaire HAL, est destinée au dépôt et à la diffusion de documents scientifiques de niveau recherche, publiés ou non, émanant des établissements d'enseignement et de recherche français ou étrangers, des laboratoires publics ou privés. 


\title{
Multi-hop wireless charging optimization in Low-Power Networks
}

\author{
Tifenn Rault, Abdelmadjid Bouabdallah, Yacine Challal \\ Université de Technologie de Compiègne \\ Heudiasyc UMR CNRS 7253 \\ 60205 Compiègne, France
}

\begin{abstract}
Recent advancements in wireless charging technology offer promising alternative to address the challenging problem of energy consumption in low-power networks. Based on these breakthroughs, existing solutions have investigated wireless charging strategies of low-power networks through the use of mobile chargers, where a charger has to come at the nodes' vicinity to recharge their battery. However, none of these works have considered the multihop energy transmission, whose feasibility have been demonstrated recently. In such a system, a node can transmit energy wirelessly to its neighbors. In this paper, we propose an optimization model to determine the minimum number of chargers needed to recharge the elements of a network in a multihop scenario, taking into acccount the energy demand of the nodes, the energy loss that occurs during a transfer and the capacity of the chargers. To the best of our knowledge, the work presented in this paper is the first that addresses the optimization of multihop wireless energy transfer in low-power networks.
\end{abstract}

\section{INTRODUCTION}

The major limitation of Low-Power and Lossy Networks (LLN) is the lifetime of the battery-powered nodes like sensors or actuators. In litterature, many energy-efficient protocols have been proposed to tackle energy consumption of lowpower nodes at all layer of the protocol stack [1], [2]. Despite these efforts, LLN lifetime remains a performance bottleneck as these solutions can only extend the network lifetime for a limited period. Similarly, energy harvesting techniques [3] have been developed to enable devices to harvest energy from their surrounding environment like sun, wind or movement. The nodes convert ambient energy to electrical energy to replenish their battery. However, energy scavenging techniques remain highly dependent on the environment as the ambient energy is not always available. Thus, the next harvesting opportunity is not easily predictable nor controllable.

In this context, recent breakthroughs in wireless energy transfer (WET) are expected to increase the sustainability of LLN and make them operational forever. For instance, a new wireless power transfer technique, called Witricity, was reported in Sciences by Kurs et al. [4]. Using Witricity, the authors were able to power a $60-\mathrm{W}$ light bulb over 2 meters with an efficiency of $40 \%$. The technique uses strongly coupled magnetic resonance to transmit power between devices without the need of any contact between the transmitter and the receiver. The applications of wireless energy transfer in lowpower and lossy networks are numerous. It has already been applied to power medical sensors and implantable devices [5], to replenish wirelessly sensors embedded in concrete [6] and to power a ground sensor from a UAV [7]. So, it is expected that wireless energy transfer will revolutionize the principles of LLN design. Indeed, the emergence of wireless power charging technology should allow overcoming the energy constraint of LLN, as it is now possible to replenish the network elements in a more controllable manner. For the moment, most of the existing works that aim to take advantage of WET consider mobile chargers that directly deliver power to deployed nodes.

A step further, Watfa et al. [8] demonstrate that it is possible to transfer wireless energy over multihop. In such a system, a device can both transmit and receive energy. This new paradigm offers unexplored perspectives regarding lifetime enhancement of LLN. Now, we can imagine that nodes are several hops away from the charger and that neighboring nodes are able to exchange energy. Inspired by this advance in multihop wireless energy transfer, we propose to optimize the locations of the chargers so that the number of chargers required to recharge all the elements of the network is minimized. Our model take into consideration the energy demand of the nodes, the energy loss that occurs during a transfer and the energy capacity of the chargers. As a result, we obtain different disjoint charging trees, so that a charger located at a root can recharge all the nodes of the charging tree.

The rest of this paper is organized as follows. In section II, we first survey studies that use wireless charging technology in LLN. We introduce the general idea of our solution in section III. Then, in section IV, we detail the proposed multihop wireless charging scheme. In section $\mathrm{V}$, we present our optimization model for multihop wireless energy transfer. We report the simulation results and discuss the advantage of our solution over single-hop energy transfer in section VI. Finally, section VII concludes the paper and provides future research directions.

\section{RELATED WORKS}

In this section, we review existing works that use wireless charging technology for lifetime prolongation in LLN. Most existing solutions consider only one-hop energy transfer through the use of mobile chargers that visit each node to recharge its battery. 


\section{A. Single-hop energy transfer}

In [9], Yao et al. propose three simple charging schemes. In two schemes, a charger is assigned to a region and computes a shortest round path which links all sensor nodes in the area. The charger patrols along this path and charges a node when it is short of energy. In the last scheme the network is not divided into regions and sensors send requests to mobile chargers when their energy falls under a given threshold. The charger closer to the sensor send a repeal packet. When a charger have to charge more than one sensor, it decides its charging sequence based on a function that takes into account the nodes residual energy and their distance to the charger. The performance metrics are the moving distance of charger nodes, the number of charge control messages and the charge latency which corresponds to the time elapsed between the moment a node send a request and the moment it is effectively recharged.

Peng et al. [10] propose a three-tier architecture composed of i) stationary sensor nodes, ii) a mobile charger (MC) and iii) an energy station that monitors the energy status of sensors and directs the mobile charger. Sensors periodically send information about their battery state, then the energy station computes a charging sequence and send commands to the MC. The authors formulate the charging problem and prove that it is NP-complete. They then present two greedy algorithms that prolong the network lifetime.

Li et al. [11] consider a mobile charger called Qi-Ferry (QiF), that must start from a charging station, visit tour stops to wirelessly charge sensors and then go back to the charging station. A tour stop is not necessarily collocated to a sensor as the QiF can charge a sensor while its distance from the sensor is less than a given threshold. The authors define the QiFerry problem which aim to maximize the number of sensors charged during a tour, while the energy spent by the mobile during the tour to move and to power sensors does not exceed its initial energy. They prove the NP-hardness of the problem and propose a PSO-based heuristic to compute a tour that covers all the sensors. Then, if the energy consumption of the QiF is not respected, the algorithm iteratively removes one tour stop that incurs the minimum reduction in coverage at a time, until the energy constraint is satisfied.

$\mathrm{Li}$ et al. [12] formulate the joint routing and charging problem for lifetime maximization (ML-JRC) and prove its NP-hardness. They give a linear programming model that determines an upper bound of the maximum network lifetime that can be achieved in the ML-JRC problem and propose three heuristic solutions. In these approaches, the time is divided into slots and at the beginning of each slot, a node selects the least-cost route to the BS by exchanging information with its neighbors. Meanwhile, the mobile charger plans its activity for this slot. Then depending on the solution, the mobile charger choses to charge either i) nodes with the minimum residual energy, ii) nodes with the minimum estimated lifetime taking into account the energy consumption rate and assuming fixed routes, and iii) nodes that bottleneck the network taking into consideration routes dynamic by solving at the beginning of each slot a modified version of the LP. In this work the MC is assumed to have a full knowledge of the network, including nodes locations and nodes energy level.

Doost et al. [13] highlight that the charging rate may be different for the nodes depending on their location-specific channel behavior. In order to enhance the network lifetime, they propose a new routing metric that favours the formation of routes including nodes that have the best energy charging characteristics. In this way, the base station selects a path with the lowest maximum charging time. The authors also consider that the wireless charging waves operates in the same frequency band as that used for communications. So, a node either receives energy or transmits packets. After selecting the optimal path, the base station runs an optimization model that determines the charging time and the transmission time of the sensors, that maximizes the thoughput under energy and latency constraints.

Shi et al [14] consider a mobile charger that periodically visits each network node to replenish their battery. They formulate an optimization model to maximize the ratio of the time spent by the charger at its home station over the time spent in charging the sensors. The authors prove that the optimal path for the charger is the shortest Hamiltonian cycle. Then, given an optimal traveling path, they formulate the joint problem for routing and charging time under the constraint that a sensor never runs out of its energy. With their approach, the network can remain operational forever, but the charger is supposed to have enough energy to recharge all sensors during a cycle.

Erol-Kantarci and Mouftah [15] propose SuReSense, a two phase algorithm for wireless rechargeable sensor networks in smart grid. The authors consider mobile chargers that can wirelessly power multiple sensors simultaneously, if the charger is located at a landmark that is in the sensors energy transfer range. The solution first runs a Linear Programming model that gives the minimum number of landmarks based on sensor energy-demand and constrained by the initial energy of the charger. Then the landmarks are grouped based on their proximity to form clusters. A mobile charger is assigned to each cluster and visite each landmark following the shortest Hamiltonian cycle.

\section{B. Multi-hop energy transfer}

Watfa et al. [8] demonstrate the feasibility of transferring energy through multihop. They achieve an efficiency of $20 \%$ over 8 hops. The authors also propose a charging strategy for a flat and a clustered topology. In the flat topology, a sensor whose energy goes below a given threshold flood a request packet in the network. If a node is able to charge the requesting sensor, it sends back a message and transmits energy (possibly along a multihop path). In a clustered architecture, the sensor first sends its request to the cluster head $(\mathrm{CH})$. If the $\mathrm{CH}$ cannot charge the sensor, it broadcast the request to the members of the cluster. If there are no node able to charge the sensor, the $\mathrm{CH}$ charge itself by sending 
requests to the other $\mathrm{CH}$.

In most of the existing solutions, authors consider only onehop wireless charging systems by using a mobile charger that must visit each node to recharge its battery. Instead, we propose to consider a multihop wireless charging scheme where a node can transmit energy to its neighbors. Particularly, we are interested in minimizing the number of chargers required to recharge the nodes of a network. This problem have not already been considered in the litterature. In the next section, we expose the general idea of our solution. We later detail the envisionned multihop wireless charging strategy and the optimization model.

\section{OVERVIEW OF OUR SOLUTION}

We consider a static wireless low-power network and a set of chargers of fixed capacity. The aim of our solution is to determine the minimum number of chargers - and their location - required to recharge nodes in a multihop scenario, taking into account the energy-demand of the nodes, the energy loss that occurs during a transfer and the capacity of the chargers. We suppose that the chargers locations are restricted to the nodes locations. As explained below, our approach employs two steps.

At the first step, for each possible location of the chargers, we construct a shortest path tree rooted at this location that covers all the nodes (using Dikjstra's algorithm). In order to take into consideration the energy losses, we consider a multiplicative cost of the edge's weight instead of an additive one. At the second step, we propose a Mixed Interger Linear Programming (MILP) model that determines the minimum number of chargers required to recharge all nodes given: the energy demand of the nodes, the energy loss that occurs during a transfer and the energy capacity of the chargers. The MILP uses the trees constructed at the first step to return the minimum number of disjoint shortest trees, so that if a charger is located at the root, it can satisfy the energy-demand of all the nodes present in the tree.

From this optimization problem, it is easy to see that if the chargers' capacity is unlimited, and because our objective is to minimize the number of chargers, our MILP will return only one tree. The drawback of this solution is the charging time and the total energy required to recharge all the nodes. On the contrary, if we suppose that the chargers have a very low energy capacity, the MILP will construct one-hop trees, so that each node will be in the vicinity of a charger. In this case, an important number of chargers will be required. Note that the recharging schedule is beyond the scope of this article. We further detail our solution in section $\mathrm{V}$, after presenting our multihop wireless charging scheme in the next section.

\section{THE MULTIHOP WIRELESS CHARGING SCHEME}

In this section, we modelize our multihop wireless charging scheme for LLN. We successively describe the network model, a one-hop energy transfer and a multihop energy transfer.

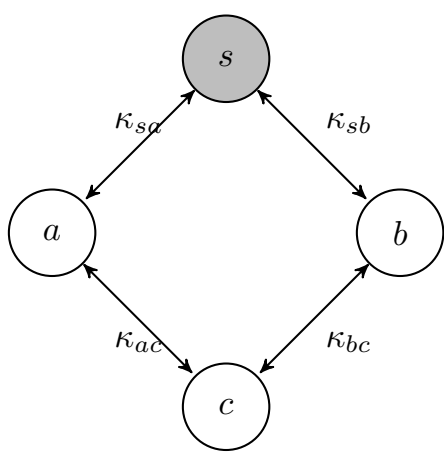

Fig. 1. A four-nodes network and their one-hop loss coefficients

\section{A. Network model}

We consider that the wireless low-power network is composed of a set $N$ of static nodes randomly deployed in a region of interest. We consider a set $S$ of chargers with identical energy capacity $C$. In our scenario, the only sources of energy in the network are the chargers. Nodes act as intermediary transmitter to transfer the energy over multihop. So, a transmitter of energy can be either a charger or a node. A receiver necessarily refers to a node. We assume that a node can receive energy from only one transmitter and that a transmitter can transmit energy to multiple neighbors, but only to one at a time ${ }^{1}$. This corresponds to a tree structure, where a node can transmit energy to its sons, and a node receives energy from its unique parent. Further, the energy-demand of a node $i$ is denoted $E_{i}>0$.

\section{B. Direct energy transfer}

We explain the one-hop energy transmission between two neighboring nodes. A transmitter $i$ can wirelessly charge one of its neighbors $j$ with a loss coefficient $\kappa_{i j} \geq 1$. This means that if the receiver needs $E_{j}$ units of energy, the transmitter must transmit $E_{j} \kappa_{i j}$ units of energy because of loss phenomenon. Technically, the energy losses of a transmission depend on the receiver and transmitter's circuitry specifities and on the distance between the two devices [8]. For the sake of simplicity, we consider that the energy transmission loss depends only on the distance between the two devices, and that the loss coefficient is an increasing function with respect to the distance. We also assume that the loss coefficient is symetric, i.e. $\kappa_{i j}=\kappa_{j i}$. We now model the network as a nonoriented graph $G(V, E)$ where $V$ is the set of static nodes. There is an edge between two nodes $i$ and $j$ if and only if they can send energy to each other. Each edge is associated a weight $\kappa_{i j}$ that corresponds to the loss coefficient of the direct transmission between $i$ and $j$. In Figure 1, we give an exemple for a four-nodes topology.

\section{Multihop energy transfer}

When the energy is transmitted from a charger to a given node through multihop path, energy is lost at each intermediate

\footnotetext{
${ }^{1}$ This assumption is justified by the fact that a receiver can switch on or off its circuit used for energy reception.
} 
transmission along this path. We denote by $P_{i j}$ the set of different paths that exist between nodes $i$ and $j$. We assume that the final loss coefficient $K_{i j}^{p}$ of a multihop transmission between a charger $j$ and a node $i$ along the path $p \in P_{i j}$, is equal to the product of the loss coefficients of the intermediate one-hop transmissions.

$$
K_{i j}^{p}=\prod_{(x, y) \in p} \kappa_{x y}
$$

Note that if there exists multiple paths from a charger to a node, the loss coefficient may be different for each path. Hence, we define $\pi_{i j}$ the minimum energy loss coefficient of a multihop transmission between a charger $j$ and a node $i$. We denote by $p_{i j}^{*}$ the path which minimizes $\pi_{i j}$.

$$
\pi_{i j}=\min _{p \in P_{i j}} K_{i j}^{p}=K_{i j}^{p_{i j}^{*}}
$$

Figure 1 illustrates a two-hops energy transmission between the charger $s$ and node $c$. Either node $a$ or $b$ can be used as an intermediate transmitter. If $a$ is used as the intermediate transmitter, to satisfy the energy-demand $E_{c}$, the charger must provide $E_{c} \kappa_{s a} \kappa_{a c}$ because of the energy loss along the path. If $b$ is selected as the intermediate transmitter, the charger will have to provide $E_{c} \kappa_{s b} \kappa_{b c}$ units of energy. Here, $\pi_{s c}=$ $\min \left(\kappa_{s a} \kappa_{a c}, \kappa_{s b} \kappa_{b c}\right)$.

\section{Charging tree}

In the graph $\mathrm{G}(\mathrm{V}, \mathrm{E})$ that we consider, we define a charging tree $T_{j}(U, A)$ as a tree rooted at $j$, so that a charger located at $j$ must supply energy to all the node $i \in U$. Moreover, the path from any node $i \in U$ to the root $j$, is the path $p_{i j}^{*}$ that minimizes the final loss coefficient $K_{i j}^{p_{i j}^{*}}$. More formally, $T_{j}(U, A)$ can be defined as follows.

$$
\begin{aligned}
& T_{j}(U, A): \\
& \left\{\begin{array}{l}
U \subseteq V \\
(x, y) \in A \text { iif }(x, y) \in E \wedge x \in U \wedge y \in U \wedge(x, y) \in p_{x j}^{*}
\end{array}\right.
\end{aligned}
$$

Clearly, there is a tradeoff between the height of a charging tree, the time required to charge all the nodes of the tree and the total amount of energy needed to cover the nodes' energy-demand. Indeed, due to the cumulative energy loss that happens during a multihop energy transfer, the longer the transmission path between the charger and the receiver is, the higher energy the charger has to provide. That's why it could be interesting to limit the maximum height $h$ of the charging trees. In order to do this, we denote by $Z_{i}^{h}$ the set of nodes that are at most $h$-hops away from $i$.

\section{THE OPTIMIZATION MODEL}

In this section, we present our optimization model for multihop wireless charging in LLN. We suppose that we have a set $S$ of chargers of identical capacity $C$. Our goal is to determine the minimum number of chargers of fixed capacity (and their locations) required to charge every element in the network through multi-hop energy transfer. Moreover, our solution ensures that the energy needed to recharge all the nodes assigned to a charger does not exceed the capacity of the charger. In what follows, we suppose that the possible locations of the chargers are restricted to the nodes locations. At the end, our solution constructs different disjoint charging trees, so that a charger located on a root can recharge all the nodes of the charging tree.

\section{Step 1: Shortest trees construction}

On the graph $G(V, E)$ that models the network, we first run a modified Dikjstra's algorithm, considering a multiplicative cost instead of an additive one. In this way, we obtain for any pair of nodes $i$ and $j$ the minimum loss coefficient $\pi_{i j}$ and the path $p_{i j}^{*}$ that minimizes this coefficient. We also compute the length of each path $p_{i j}^{*}$, and we denote by $l\left(p_{i j}^{*}\right)$ the number of intermediate nodes of the path between $i$ and $j$. So, if $i$ and $j$ are two neighboring nodes, $l\left(p_{i j}^{*}\right)=0$. The two quantities $\pi_{i j}$ and $l\left(p_{i j}^{*}\right)$ are then used as parameters of the MILP in the second step.

\section{Step 2: Optimization of the number of chargers}

In the second step, we run a Mixed Integer Linear Programming (MILP) model that minimizes the number of chargers under several energy constraints. Indeed, our solution takes into account the energy-demand of the nodes, the energy capacity of the chargers and the cumulative energy loss that occurs during multihop energy transfer. Moreover, we can bound the maximum height of the charging trees by $h$, i.e. energy is not transmitted over more than $h$ hops. We recall that $Z_{i}^{h}$ denotes the set of nodes that are at most $h$-hops away from $i$. Our MILP uses a binary variable $B_{i j}\left(i \in N, j \in Z_{i}^{h}\right)$, that is equal to 1 if the node $i$ belongs to the charging tree $T_{j}$, and 0 otherwhise. More specifically, $B_{j j}$ is equal to 1 if and only if the charging tree rooted at $j$ exists. We can now formulate the MILP that minimizes the number of chargers as below:

$$
\begin{gathered}
\min \sum_{j \in N} B_{j j} \text { subject to } \\
B_{i j} \leq B_{j j}, \quad i \in N, j \in Z_{i}^{h} \\
\sum_{j \in Z_{i}^{h}} B_{i j}=1, \quad i \in N \\
\sum_{i \in Z_{j}^{h}} E_{i} \pi_{i j} B_{i j} \leq C, \quad j \in N \\
\sum_{x \in P_{i j}} B_{x j} \geq l\left(p_{i j}^{*}\right) B_{i j}, \quad i \in N, j \in Z_{i}^{h}, i \neq j \\
B_{i j} \in\{0,1\}, \quad i \in N, j \in Z_{i}^{h}
\end{gathered}
$$

The objective function (4) minimizes the number of chargers, i.e. the sum of nodes that are selected as roots. Constraint (5) ensures that a node $i$ cannot belong to a tree rooted at $j$ if the node $j$ is not selected as a root. In other words, 
the tree rooted at $j$ does not exist. Constraint (6) guarantees that a node has only one charger. It also assures that a node belongs to only one tree. Constraint (7) refers to the chargers' limited energy capacity. The left part of the inequality in the constraint represents the amount of energy a charger located at node $j$ must provide to satisfy the energy demand of all the nodes belonging to the tree rooted in $j$. The right part of the inequality specifies that the amount of energy a charger will have to provide must not exceed its capacity. Constraint (8) ensures that we obtain disjoint charging tree. Indeed, (8) states that a node $i$ cannot belong to a tree rooted at $j$ if the intermediate nodes involved in the transmission of the energy from $j$ to $i$ (i.e. the nodes that belong to the optimal path $p_{i j}^{*}$ ) are not in the tree $j$. Finally, constraint (9) states that $B_{i j}$ are binary variables.

\section{PERFormance EVALUATION}

The network that we consider for performance evaluation is composed of 100 nodes randomly deployed in a rectangular field of $100 \mathrm{~m} \times 100 \mathrm{~m}$. The energy-demand $E_{i}$ of every node is set to $1 \mathrm{KJ}$. We vary the battery capacity of the chargers between $20 \mathrm{KJ}$ as in [15] and 2000KJ as in [10]. We also vary the maximum height of the charging trees from 1 to 6 hops. Two neighboring nodes can exchange energy if there are at most 15 meters away from each other. The loss coefficient between two neighboring nodes is set equal to the euclidian distance that separates the two nodes. We solved the MILP models with CPLEX ${ }^{2}$ and the constraints are generated in $\mathrm{C}++$. In what follows, we study for different values of parameters, the optimal number of chargers and the total energy necessary to satisfy the energy-demand of the nodes. Note that the maximum height of the charging trees corresponds to the parameter $h$ of the MILP, which means that the energy is transmitted at most over $h$-hops (the height of the charging trees is at most $h$ ). This does not mean that the obtained charging trees have a height equal to $h$. Indeed, even if the solver will tend to construct charging trees with high height in order to minimize the number of chargers, if the battery capacity of the chargers is too low, the chargers will only be able to supply energy to smaller trees.

Figure 2 shows the minimum number of chargers required to satisfy the energy-demand of the nodes depending on the maximum height of the charging trees and the battery capacity of the chargers. We can make several observations. First, as expected, higher is the chargers's battery capacity, smaller is the number of chargers. This is because a charger will be able to serve more nodes. Second, we can see that the maximum height of the charging trees and the chargers' capacity highly constrain the problem. Indeed, for every battery capacity, after 3 hops, the minimum number of chargers does not change. This is because the energy capacity of the charger is not sufficient to build charging trees with more hops. Thus, for every capacity, after 3 hops, the solution does not change any

\footnotetext{
${ }^{2} \mathrm{http} / /$ www-01.ibm.com/software/integration/optimization/cplexoptimizer/
}

more and the resulting networks are similar. Third, our results bring useful information regarding the network dimensioning. When the battery capacity of the chargers increases from $20 \mathrm{KJ}$ to $100 \mathrm{KJ}$, we cut the number of chargers by two. But when it passes from $500 \mathrm{KJ}$ to $2000 \mathrm{KJ}$, we only save three chargers. So, we may prefer to use 10 chargers of $500 \mathrm{KJ}$ instead of 7 chargers of $2000 \mathrm{KJ}$, even if it is not the optimal solution.

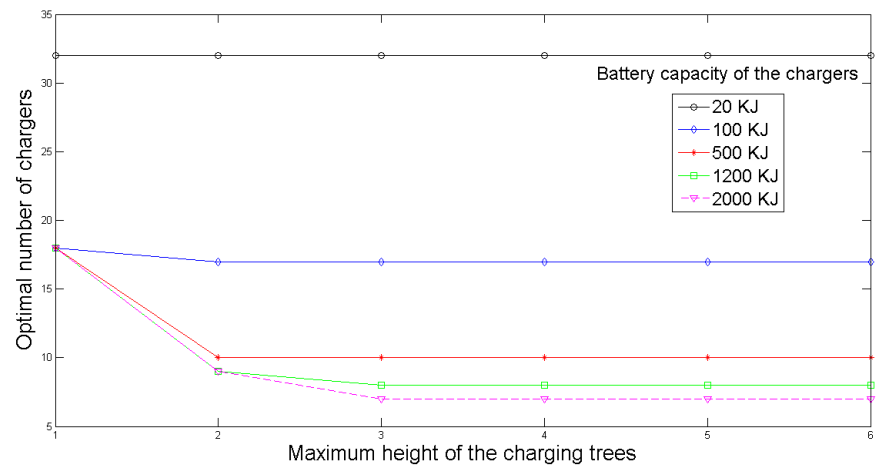

Fig. 2. The minimum number of chargers depending on the maximum height of the charging trees and the battery capacity of the chargers.

From another point of view, Figure 3 compares the simulation results of our multihop energy transfer scheme to a naive single-hop energy transfer approach, which is similar to [15], where the parameter $h$ is set to 1 . As expected, our solution enables to decrease the total number of chargers required to satisfy the energy-demand of every node. This is because an higher tree can cover more nodes. The only exception is when the chargers capacity is very low $(20 \mathrm{KJ})$, as the minimum number of chargers is 32 , whatever is the considered approach. In this case, the energy is so low that chargers can only supply energy to the nodes that are one-hop away from them. So, even though we increase the maximum authorized height, it is not possible to decrease the number of chargers by building higher trees. Regarding the network dimensioning, we can observe that if one of the requirement is to construct 1-hop charging trees, the minimum number of chargers is 18 . In this special case, it is not necessary to use chargers with more than $100 \mathrm{KJ}$ as it will not improve the solution.

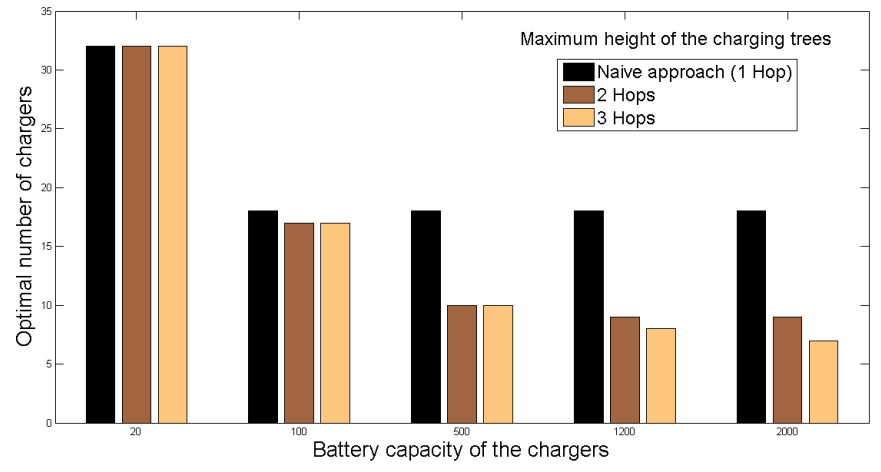

Fig. 3. The minimum number of chargers for our multihop energy transfer scheme compared to a naive single hop energy transfer approach.

The amount of energy a charger located at node $j$ must 
provide to satisfy the energy demand of all the nodes belonging to the tree rooted at $j$ is equal to $\sum_{i \in Z_{j}^{h}} E_{i} \pi_{i j} B_{i j}$ as explained for the constraint (7). We call this quantity the energy-supply of a charger. We define the total energy supply as the sum of the energy-supply of each charger. The minimum total energy supply we can obtain is $100 \mathrm{KJ}$ when a mobile charger is assigned to every node. In this case, a charger has to supply $1 \mathrm{KJ}$ to its single node and we have hundred charging trees of height equal to 0 . This gives a total energy of $100 \mathrm{KJ}$. Even if this solution minimizes the total energy supply, it is not conceivable as it requires one charger per node.

Figure 4 represents the total energy supply required to satisfy the energy-demand of the nodes depending on the maximum height of the charging trees and the battery capacity of the chargers. When the height of the charging trees increases, the total energy supply also increases. This is expected as the cumulative loss coefficient increases when the number of hops that separates the node from the charger increases. From Figure 4, we see that for every battery capacity, after 3 hops, the total energy supply does not change. This can be explained by the fact that the optimal number of chargers does not change after 3 hops as we have seen in Figure 2.

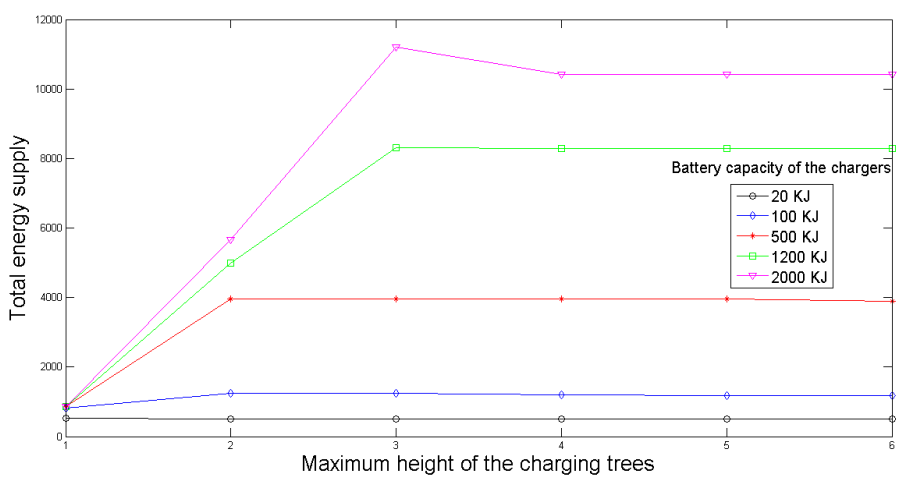

Fig. 4. The total energy supply, depending on the maximum height of the charging trees and the battery capacity of the chargers.

\section{CONCLusion AND Future Directions}

In this paper, we have explored how multihop wireless energy transfer paradigm can be used to enhance the lifetime of low-power networks, by charging nodes in a more controllable manner. In particular, we have developped an optimization model to minimize the number of required chargers while taking into account the energy-demand of the nodes, the cumulative energy loss that occurs during a transfer and the capacity of the chargers. As expected, multihop energy transfer enables us to reduce the number of chargers necessary to satisfy the energy-demand of every node. Furthermore, we highlighted that there is a tradeoff between the number of chargers, the height of the charging trees and the total amount of energy needed to cover the nodes' energy-demand over multihop.

Future directions include considering that a transmitter can transfer energy to multiple receivers at a time. The wireless energy transfer to multiple receivers has aldready been considered in WSN [16], [17], but never in a multihop scenario. This will lead to more complex wireless energy transfer schemes but will certainly require less energy. Another issue that has not been addressed in this paper is the recharging schedule. Indeed, once the chargers are deployed, it could be interesting to find out a schedule that minimizes the charging latency.

\section{AKNOWLEDGMENTS}

This work was carried out and funded in the framework of the Labex MS2T. It was supported by the French Government, through the program "Investments for the future" managed by the National Agency for Research (Reference ANR-11-IDEX0004-02).

\section{REFERENCES}

[1] K. Akkaya and M. Younis, "A survey on routing protocols for wireless sensor networks," Ad Hoc Networks, vol. 3, no. 3, pp. 325-349, 2005.

[2] I. Demirkol, C. Ersoy, and F. Alagoz, "MAC protocols for wireless sensor networks: a survey," IEEE Communications Magazine, vol. 44, no. 4, pp. 115-121, 2006.

[3] S. Sudevalayam and P. Kulkarni, "Energy Harvesting Sensor Nodes: Survey and Implications," IEEE Communications Surveys \& Tutorials, vol. 13 , no. 3, pp. 443-461, 2011.

[4] A. Kurs, A. Karalis, R. Moffat, J. Joannopoulos, P. Fisher, and M. Soljacic, "Wireless power transfer via strongly coupled magnetic resonances," Science, vol. 317, pp. 83-86, 2007.

[5] F. Zhang, S. A. Hackworth, X. Liu, H. Chen, S. R. J., and M. Sun, "Wireless energy transfer platform for medical sensors and implantable devices," IEEE Int. Conf. on Engineering in Medicine and Biology Society - EMBS, pp. 1045-1048, 2012.

[6] O. Jonah and S. V. Georgakopoulos, "Efficient wireless powering of sensors embedded in concrete via magnetic resonance," IEEE Int. Symposium on Antennas and Propagation, pp. 1425-1428, 2011.

[7] B. Griffin and C. Detweiler, "Resonant wireless power transfer to ground sensors from a UAV," IEEE Int. Conf. on Robotics and Automation, pp. 2660-2665, 2012.

[8] M. K. Watfa, H. Al-Hassanieh, and S. Salmen, "The road to immortal sensor nodes," Int. Conf. on Intelligent Sensors, Sensor Networks and Information Processing, pp. 523-528, 2008.

[9] W. Yao, M. Li, and M.-Y. Wu, "Inductive charging with multiple charger nodes in wireless sensor networks," Int. Conf. on Advanced Web and Network Technologies, and Applications, pp. 262-270, 2006.

[10] Y. Peng, Z. Li, W. Zhang, and D. Qiao, "Prolonging sensor network lifetime through wireless charging ," IEEE Real-Time Systems Symposium - RTSS, pp. 129-139, 2010

[11] K. Li, H. Luan, and C.-C. Shen, "Qi-Ferry: energy-constrained wireless charging in wireless sensor networks ," IEEE Wireless Communications and Networking Conference: Mobile and Wireless Networks, pp. 25152520, 2012.

[12] Z. Li, Y. Peng, W. Zhang, and D. Qiao, "Study of joint routing and wireless charging strategies in sensor networks " Proceedings of the 5th Int. Conf. on Wireless algorithms, systems, and applications, pp. $125-135,2010$.

[13] R. Doost, K. R. Chowdhury, and M. Di Felice, "Routing and link layer protocol design for sensor networks with wireless energy transfer ," IEEE Global Telecommunications Conference - GLOBECOM, pp. 1-5, 2010.

[14] Y. Shi, L. Xie, Y. T. Hou, and H. D. Sherali, "On renewable sensor networks with wireless energy transfer," IEEE Int. Conference on Computer Communications - INFOCOM, pp. 1350-1358, 2011.

[15] M. Erol-Kantarci and H. T. Mouftah, "SuReSense: sustainable wireless rechargeable sensor networks for the Smart Grid," in IEEE Wireless Communications, vol. 19, no. 3, 2012, pp. 30-36.

[16] L. Xie, Y. Shi, Y. T. Hou, W. Lou, H. D. Sherali, and S. F. Midkiff, "On renewable sensor networks with wireless energy transfer: the multi-node case," IEEE Int. Conference on Sensor, Mesh and Ad Hoc Communications Networks - SECON, pp. 10-18, 2012.

[17] B. Tong, Z. Li, G. Wang, and W. Zhang, "On renewable sensor networks with wireless energy transfer: the multi-node case," IEEE Int. Conference on Distributed Computing Systems - ICDCS, pp. 438-447, 2010. 